\title{
Un milagro de San Isidro relacionado con ritos de protección del grano durante la siembra
}

En muchas ocasiones la ausencia de documentación de carácter histórico hace que la antigüedad de determinadas prácticas populares deba ser únicamente una mera suposición basada en la lógica. A veces, no obstante, encontramos textos que permiten fijar de manera precisa la perduración a través del tiempo de una serie de costumbres que en nuestros dias son residuales o han desaparecido. Sin intentar resolver lo complicado del fenómeno que supone la constatación de ritos similares en épocas y países alejados entre sí, aunque con una cierta proximidad geográfica, vamos a analizar una noticia sobre lo que fue un rito protector realizado durante la siembra de los cereales, así como diversas actitudes ante los animales que pueden resultar dañinos para el éxito de la cosecha en el momento de la siembra.

El tema que da pie a este trabajo es uno de los milagros atribuidos a San Isidro; milagro que podríamos calificar de "nuevo", ya que es de los que aparecen en el siglo xvir en textos eruditos y cuyo origen hay que buscar en los procesos de beatificación y canonización que se desarrollan desde finales del siglo XVI hasta $1622^{1}$. Por lo general, estas narraciones suelen tener bastante relación con los milagros que aparecen recogidos en el códice de Juan Diácono, del siglo xuI, aunque siempre suponen una clara alteración de los mismos ${ }^{2}$.

\section{EL MILAGRO DE SAN ISIDRO}

En los textos del siglo XVII vemos cómo el número de milagros atribuidos al santo aumenta, produciéndose, a la vez, la repetición de los ya conocidos,

1 Sobre este tema hemos hablado en nuestro trabajo "Ángeles labradores y lamias: una visión mítica del trabajo agricola", Primeras Jornadas internacionales sobre tecnología agraria tradicional (Madrid: Ministerio de Cultura, en prensa).

2 Puede verse en su versión latina en Fidel FITA -Año 1275. Leyenda de San Isidro por el diácono Juan.-Códice del siglo xIII, procedente del archivo parroquial de San Andrés., Boletín de la Real Academia de la Historia, IX (1886): 97-157. Ha sido traducido recientemente por María Paz HERRERo LORENZO, Los milagros de San Isidro. Códice de Juan Diácono (Madrid: s.e., 1988). 
que pasan a convertirse casi en actos cotidianos. Esta actitud puede explicarse tanto por la incidencia de la tradición oral - que sitúa el mismo milagro en distintos lugares - como por la pretensión de magnificar la figura del santo incrementando el número de acciones milagrosas. Ambas corrientes se unen en la documentación de los procesos de beatificación y canonización y se difunden posteriormente en las numerosas biografias que se escriben a raíz de su elevación a los altares.

La versión más antigua que hemos localizado procede del libro que Gerónimo de Quintana publica sobre Madrid en 1629. Es característico de este autor la ausencia de rigor, que contrasta con la prudencia que Jaime Bleda muestra por esa misma época, por ejemplo ${ }^{3}$. Los hechos, tal como los narra Quintana, suceden así:

Auia llegado el nuestro [santo] a este estado desta soberana simplicidad, o por mejor dezir suma sabiduria, y era tan sencillo, que quando salia al campo a su labor, no solo repartia con los pobres lo que encontraua del trigo que lleuaua para sembrar, sino que tambien echaua dello a puñados a las aues diziendo: Tomad auecicas de Dios, que quando Dios amanece, para todos amanece. Con lo qual se desmenguauan gran parte los costales, mas como lo hazia con tan recta intencion, sin animo de defraudar a su dueño en cosa alguna, mouido tan solamente dela compassion que tenia de las necessidades agenas, y de la caridad que le gouernaua, la soberana Magestad (cuyo es el dominio absoluto detodas las criaturas,) en demostracion de que se auia seruido de aquel hecho, y que la propiedad del trigo la auia transferido a quien nuestro Santo la auia repartido, ordenò que quando llegasse a la heredad no le faltasse grano, hallando los costales tan llenos como los auia sacado de su casa. Quedò nuestro ISIDRO confuso y suspenso, y encomendandolo al silencio, agradecido, y con nueua confiança, quando empeçaua a sembrar dezia, arroxando el trigo: En nombre de Dios, esto para Dios, y esto para nos, esto para las aues, y esto para las hormigas. Los labradores circunuezinos que estauan a la mira, viendo lo que passaua, y oyendo lo que dezia, con vna falsa disimulacion de burla y mofa que hazian del, dentro de si teniendole por tonto y mentecato, le preguntauan y tambien para las hormigas? El Santo lleno de gozo, y embeuida su memoria en la passada marauilla, respondiò con su senzillez y mansedumbre acostumbrada. Si, que para todos dà Dios*.

Si en lugar de encontramos al santo yendo camino a la heredad para sembrar, se hubiera sustituido el escenario por el transporte del grano al

3 Como indicamos en nuestro trabajo citado en la nota 1, Quintana incluye narraciones con un fuerte sabor popular $y$, en parte, mágico, que es posible documentar aún en el ámbito rural hispano.

- Gerónimo de QUINTANA, Historia de la antigüedad, nobleza y grandeza de la villa de Madrid,... (Madrid, 1629), fol. 118 v. Existe edición facsímil en dos tomos (Madrid: Marcos Real Editor, 1986). Para facilitar los trabajos de imprenta optamos por desarrollar las abreviaturas existentes en los textos antiguos. 
molino nos hallaríamos ante uno de los milagros clásicos de San Isidro, que aparece ya representado iconográficamente en el arca de finales del siglo XIII. No obstante, la narración de Quintana no deja lugar a dudas ya que, más adelante, relatará el milagro del molino por dos veces ${ }^{5} \mathrm{y}$, además, alude a la denuncia de los labradores ante el patrón del santo por el desperdicio de grano que supone el dárselo a los pájaros y a las hormigas:

...le dixeron que su criado ISIDRo era vn haragan y perdido, yendo tarde a la labrança, y que en perjuyzio y daño suyo, con piedad necia y loca, quando iba al campo arrojaua todo el trigo que lleuaua para sembrar, a las aues, haziendo otras cosas agenas de hombre de buen seso, y propias de persona loca, como el lo era, pues assi desperdiciaua su hazienda ${ }^{6}$.

Esta acusación también encuentra su paralelo en la que da lugar a la constatación del milagro de los ángeles que aran junto a Isidro pero, al contrario de lo que sucede allí, en el caso que nos ocupa, el patrón apoya la actuación del santo, confiando plenamente en su comportamiento.

Pocos años después de la edición del libro de Quintana, nos encontramos con otro texto mucho más interesante desde nuestro punto de vista, ya que enriquece la narración anterior añadiéndole una explicación sobre su procedencia y mencionando un acto similar ocurrido en otro momento y lugar. Gregorio de Argaiz, en su biografía del santo, dice:

Quando salia a sembrar no solo gozauan de su liberalidad las aues del ayre, sino las hormigas de la tierra. No se de donde hallò Geronimo de Quintana vnas palabras, que pone como texto de este Santo, que las dezia estando en el campo. Tomad auecitas de Dios, que quando Dios amanece, para todos amanece. Y quando echaua los granos en la heredad. En nombre de Dios esto para nos, esto para las aues del ayre, $y$ esto para las hormigas. Todo vendria por tradicion: $y$ asy las creo: porque en la boca de los buenos y Christianos labradores, son muy comunes, y antiguas,... ?

A continuación menciona otro hecho a destacar, el que esta frase también se decía en La Rioja, tal como se recoge en una historia del monasterio de Valvanera: "Otras palabras como estas dichas por otro labrador, y oidas de aquel salteador Munio; que tenia assombrados los moradores del Distercio

5 G. de QuINTANA, op. cit. en nota anterior, fols. 119r-119v y 132r-132v. En esta ocasión varía ligeramente el ofrecimiento a las aves: -Venid auecicas de Dios y comed, que quando Dios amanece para todos amanece. (el subrayado es del autor).

6 G. de QuinTana, op. cit. en nota 4 fol. $120 \mathrm{v}$.

7 Gregorio de ARGAIZ, La soledad y el campo laureados por el solitario de Roma, y el labrador de Madrid, San Benito y San Isidro. Dedicados por el M. Fr. Gregorio de Argayz, Chronista de su Religion, al glorioso padre San Benito, Patriarca del Occidente, y grande bijo de la iglesia (Madrid: Francisco Nieto, 1671), $18+242+21$ pp., p. 135. 
en la Rioja, bastaron para conuertirle de su mala vida, y hazerse santo, como se halla escrito en la historia del Santuario de Balbanera, fiandole Dios el descubrimiento de la escondida imagen de su Madre ${ }^{8}$. La alusión a la procedencia popular, la tradición, y el hecho de que sson muy comunes, y antiguas" son interesantes, ya que nos ponen en el camino de la correcta interpretación del entorno que da origen al milagro.

En biografías posteriores se recurre a copiar el texto de Quintana, como sucede con Raphael Ortiz ${ }^{9}$, o el de Argaiz, como ocurre con N. J. de la Cruz. Es interesante resaltar que este último autor parece dar a entender que esta práctica no le es familiar, ya que dice de forma expresa: "quizá sería costumbre antigua en los labradores virtuosos", y añade que cada vez que Isidro hacía alusión a Dios, a él mismo, a las aves y a las hormigas, sembraba un puñado de grano ${ }^{10}$. Ambos matices: el considerar que sería una práctica antigua y el mencionar que el puñado era "sembrado" están indicando, en nuestra opinión, posiblemente, el abandono generalizado del mismo, si tenemos en cuenta las palabras que Argaiz dice sobre su difusión.

Hay que aclarar que el milagro no aparece en el Flos sanctorum de Alonso de Villegas durante el siglo XVI —en las ediciones de 1584, 1589, 1594 ó 1599 debido a que Isidro aún no estaba canonizado, pero sí se recoge en ediciones del XVII, copiando el Flos sanctorum de Pedro de Ribadeneyra ${ }^{11}$. En este texto la alusión al milagro de la siembra es mínima, ya que tras narrar el aumento del grano en los costales después de haber sido repartido entre los pobres y las aves, considera normal que el santo hiciera lo mismo al ir a sembrar o al molino en varias ocasiones. En el reparto previo a la siembra decía solamente: «En nombre de Dios, esto para Dios, esto para nosotros, y esto para las hormigas" ${ }^{12}$.

8 G. de ARGaIZ, op. cit. en nota anterior, p. 135.

9 Raphael ORTIZ, Vida, y milagros del glorioso San Isidro Labrador, parron (sic) de la Coronada Villa de Madrid. Reimpresa nuevamente por un Devoto... (Madrid: Por Francisco del Hierro, 1723), $190 \mathrm{pp}$. Incluye una censura y una licencia fechadas en 1713, además de la licencia de $1722,190 \mathrm{pp}$. No alude a la leyenda del santuario de Valvanera; su texto, en el que se citan a Dios, al labrador, a las aves y a las hormigas, está en las pp. 33-35.

1" Nicolás Joseph de la CRUZ, Vida de San Isidro labrador patron de Madrid, adjunta la de su esposa Santa María de la Cabeza: escrita por el R. P. Fr. ... (Madrid: Imprenta Real, 1790, 2. ${ }^{a}$ ed.), p. 25. Hay una edición facsímil (Madrid: Marcos Real Editor, 1986), $328+52$ pp. de un Apéndice.

"El texto de Alonso de Villegas correspondiente a San Isidro se puede ver, con la grafía modemizada, en Ana MARTINEZ ARANCÓN, Santoral extravagante. Una lectura del Flos Sanctorum de Alonso de Villegas (Madrid: Editora Nacional, 1978), 430 pp., en concreto en la p. 410. Se ha tomado como fuente una edición barcelonesa de 1775 que, al parecer, su editora supone la original.

12 Pedro de Ribadeneyra, Flos sanctorum. Tercera parte. En que se contienen las vidas de los Santos pertenecientes a los meses de mayo, y junio incluidas otras vidas de Santos. 


\section{El. MILAGRO DE LA VIRGEN DE VAlVANERA (LA RIOJA)}

En las historias sobre el hallazgo de la imagen y los milagros de la Virgen de Valvanera que hemos podido consultar no aparece la costumbre tal como se narra en el milagro del santo madrileño, a pesar de que el comentario de Argaiz no deja lugar a ninguna duda. Posiblemente la explicación de esta ausencia debe encontrarse en la existencia de un libro inédito de $\mathrm{D}$. Antonio de Nobis -conocido también como Antonio de Lupián Zapata o Lupián de Zapata-, Historia del santuario de Santa María de Valuanera. Este autor, conocido falsario, era amigo de Argaiz, quien, probablemente, debió consultar el manuscrito original, en el que aparecería la ofrenda a los pájaros y a las hormigas ${ }^{13}$.

A pesar de esta diferencia, el comportamiento del labrador riojano, que conduce a la conversión del bandido Nuño, o Munio Oñez, es interesante, porque también hace un ofrecimiento del grano cuando va a sembrar. Francisco de Ariz, en su obra, publicada en 1608, narra el acontecimiento de la siguiente forma:

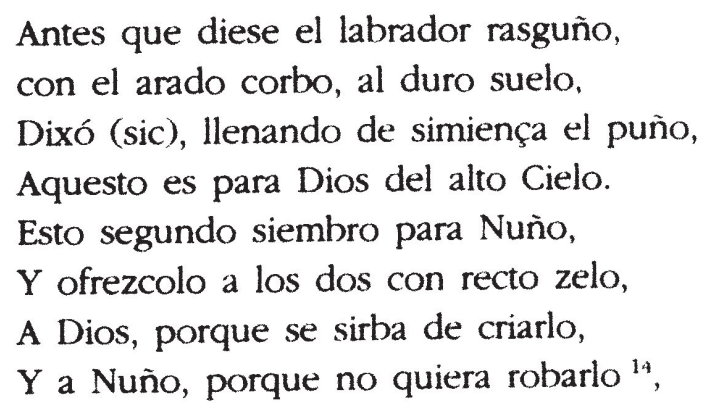

Este ofrecimiento, oído por Nuño que se había ocultado con la intención de robar los bueyes y el grano al labrador, le hace cambiar drásticamente su conducta y le lleva a convertirse en ermitano. Destaquemos como hecho interesante, por lo que se verá luego, que una parte del grano se ofrece a un ladrón, con el fin de que no robe la cosecha una vez crecida. Esta forma de actuar podría ponerse, sin mucho problema, en relación con el compor-

escritas por el V. P. Juan Eusebio Nieremberg y Padre Francisco Garcia... (Madrid: Imprenta de Agustín Fernández, 1716), p. 175. La vida de San Isidro está en las pp. 171-190.

13 La amistad es señalada por Alejandro PÉREZ Alonso, en Historia de la Real Abadía de Nuestra Señora de Valvanera en La Rioja (Oviedo: s.e., 1971), en p. 13. Además de la bibliografía que cita sobre Lupián de Zapata, se puede consultar el libro de Julio CARO BAROJA, Las falsificaciones de la Historia (en relación con la de España) (Barcelona: Seix Barral, 1992), pp. 99-102, donde se dice que Argaiz publicó el falso cronicón de Hauberto, escrito por Lupián de Zapata, precisamente en el año de muerte de éste.

14 Francisco de ARIZ, Historia de la Antiquisima Imagen de Nuestra Señora de Valvanera, y por quien fue hallada. en los años 360 ... Por su criado el Capitan D. ... (Alcalá: por Luys Martinez Grande, 1608), 124 fols, en fol 21v. 
tamiento mostrado ante pájaros y hormigas que, en cierta medida, son considerados ladrones del grano de la cosecha futura, y del que se siembra en ese momento.

En 1610, Gregorio Bravo de Sotomayor ofrece una versión totalmente diferente. En esta ocasión, en la oración que hace pide que la cosecha sea buena para que llegue a los ministros de la iglesia - a través del diezmo, suponemos - y a los pobres; quedan, por lo tanto, fuera del reparto las aves y las hormigas y, además, se excluye al ladrón que está observando la siembra:

O piadosissimo Señor, Criador del Cielo, y de la tierra, que todo lo criastes de nada: sed servido que la semilla que en esta heredad siembro, de tal manera produzga (sic), viua y crezca, que con el fruto que della se cogiere, puedan largamente lleuar los ministros de vuestra Yglesia su parte, vuestros pobres la suya, y para que a los buenos y malos, no falte el necessario sustento, por que desta suerte vuestro santo nombre sea loado y bendito de tododos (sic) para siempre jamas. Amen ${ }^{15}$.

La misma plegaria es recogida, más tarde, en la obra de Diego de Sylva y Pacheco de forma bastante literal ${ }^{16}$, mientras que la alusión a la familia del

15 Gregorio BRAvo DE SOTOMAYOR, Historia de la imbencion, fundacion. y Milagros de nuestra Señora de Valvanera, de la Orden de S. Benito. Compuesta por el Padre Fray ... (Logroño: Iuan de Mongaston Impresor de libros, 1610), 162 fols., en fols. 8v-9r. La ofrenda tiene precedentes no publicados hasta el siglo $\mathrm{XX}$ pero que estuvieron al alcance de los historiadores del santuario. Nos referimos a la denominada Historia latina, que no es sino una traducción al latín hecha en 1419, por el abad del santuario don Domingo de Castroviejo, de un texto que debió ser escrito por Gonzalo de Berceo a mediados del siglo XIII y que en la actualidad no se conoce; en su versión latina dice: In dei nomen amen. O pijssime deus celi et terre plasmator qui cuncta ex nichilo creasti. istud genus seminis quod in terram proicio. fac que so germinare. uiuere. crescere. et ad messem cum multiplici fructu perducere dignare. taliter quod ex fructu inde colligendo. ministri ecclessie tue porcionem suam accipiant. et pauperes alimonijs'nutriantur. ac eciam boni et mali decenter sustententur. ut sic ab omnibus nomen sanctum tuum laudetur et benedicatur in sempiternum. amen, cuya traducción es: .En el nombre de Dios, amén. Oh Dios piadosísimo, Creador del cielo y de la tierra, que sacaste todas las cosas de la nada. Haced, os ruego, que esta semilla que entrego a la tierra, germine, viva y crezca y os digneis llevarla a la perfección con mucho fruto; de tal modo, que de él perciban su parte los ministros de la Iglesia, se alimenten los pobres, y los buenos y malos tengan también decente sustento, para que así tu santo nombre sea alabado y bendecido por todos eternamente amen, A. PÉrEz Alonso, op. cit. en nota 13, pp. 466 y 504, respectivamente; en las pp. 441-461 hace el estudio crítico de esta Historia.

16 Fray Diego de SYLVA Y PACHECO, Historia de la Imagen sagrada de Maria Santissima de Valvanera, en el oriente de su bermosura en los Montes Distercios y eclypsi de sus luces en in roble basta la plenitud de sv candor en la aurora. que se descubrio en sv ballazgo... Dedicala... (Madrid: Imprenta de San Martin, 1665), 254 fols. + índices, en fol. 70v. 
labrador es el único cambio que se puede apreciar en la obra que fray Benito Rubio publica en 1761: que con su cosecha pueda lograr yo sustentar mi familia, los Ministros de la Iglesia, y los Pobres, y con la Bendicion de vuestra misericordia no falte à nadie el sustento.... ${ }^{17}$.

\section{UNA EXPLICACIÓN: LA REINTERPRETACIÓN DE UN RTTO DE SIEMBRA}

Acudiendo al comentario de Argaiz, e independientemente de que se llevara a cabo en La Rioja, sacamos la impresión de que el comportamiento adjudicado a San Isidro debía ser normal en buena parte de los labradores españoles del siglo xvir. No podemos, sin embargo, delimitar el área de extensión en la que se practicaba tal costumbre que, por otra parte, no tiene por qué ser homogénea. Si recurrimos a datos etnográficos publicados en el presente siglo comprobamos que la extensión geográfica de este uso en nuestro país se amplía de forma considerable. Carré Alvarellos, en un artículo sobre la zona coruñesa de la terra das Mariñas, menciona una práctica absolutamente igual a la que hemos visto asociada a San Isidro y cuya finalidad era proteger el grano sembrado ${ }^{18}$. Antes de la siembra se conjuraba a los pájaros, lo cual podía hacerse de dos maneras: sembrando un terreno especial para ellos o mediante el enchavellado. Esta segunda práctica consistía en que el sembrador echaba grano por los orificios del timón del arado, siendo recogido por la persona que conducía a los animales y entablándose entre ambos el siguiente diálogo:

- "Qué sementas labrador?"

- "Trigo vou a sementar, e paxaras a embutillar (poñerles un butillo ou buzo), paxaras embutillo, paxaros embutillo"

y posteriormente se dirigía a la tierra y echaba un puñado no arredo, zona que bordea al campo y que no es posible sembrar, y otro en la leira, terreno cultivado, diciendo: “este pra vós, e este pra nós". El reparto, según Carré

17 Fr. Benito RUBIO, Historia del venerable y antiquissimo santuario de nuestra Señora de Valvanera, en la provincia de La Rioja: felicissimo ballazgo de la celestial Imagen de Maria santissima en los Montes Distercios: su culto, y veneracion en la Orden de San Benito... (Logroño: Imprenta de Francisco Delgado, 1761), 176 pp. + índices, en p. 28.

18 Loís Carre Alvarellos, sabores da terra - O trigo, Actas do Coloquio de Estudos Etnográficos "Dr. José Leite de Vasconcellos", vol II, pp. 75-81 (Porto: Junta de Provincia do Douro Litoral, 1959). El rito se describe en las pp. 77-78. Además, en esta zona se conservaba otra costumbre relacionada con la siembra del trigo; si el dueño de un terreno encargaba este trabajo a otra persona, debía, al menos, sembrar una pequeña porción del terreno por su propia mano previamente a que aquella realizara la labor. 
Alvarellos, suponía incluir no sólo a los pájaros, sino también a los ratones y a todos los animales que pudieran comerse el grano.

Otros labradores, al sembrar la primera leira, decían: "pra Dios e pra nós" y al echar la simiente alrededor continuaban: "pra os paxaros, pra os ratos e mais pra os ladros".

Como se aprecia, la similitud es absoluta, si se exceptúa el detalle de la exclusión de las hormigas en el reparto y, en el segundo caso, de la inclusión de los ladrones; aunque ésta tiene su paralelo en la narración del milagro de la Virgen de Valvanera. Los datos que proporciona el autor gallego permiten, por lo tanto, comprender mejor lo que se narra en el milagro de San Isidro y corregir la intencionalidad originaria del acto descrito. Este acto lo que hace es mostrarnos cómo se ha reinterpretado o explicado un comportamiento campesino desde una perspectiva católica y que, al igual que sucede en Galicia, aparece cristianizado, ya que se incluye el ofrecimiento de parte de la cosecha a Dios.

Los datos que poseemos de Cataluña nos muestran un mayor grado de variedad a la hora de hacer el ofrecimiento, incluyendo una versión totalmente cristianizada del mismo. El más cercano al milagro de San Isidro procede de San Cugat del Vallès (Barcelona) y en él los gusanos, cuques $^{19}$, y la niebla, neula, aparecen como receptores de grano, junto a los pájaros y el campesino, mostrando así una posibilidad no constatada hasta ahora. Fue recogido por J. Amades, quien no ofrece más precisión que el texto siguiente, que era cantado:

$$
\begin{aligned}
& \text { Un pels ocells } \\
& \text { un per les cuques } \\
& \text { un per la neula } \\
& \text { i un pel pagès } 20
\end{aligned}
$$

Otros ritos se asemejan en parte a lo ya descrito por la existencia del reparto, aunque en esta ocasión junto a la inclusión de otros elementos naturales en el ofrecimiento del sembrador aparece un factor nuevo: los posibles restos de un sacrificio sangriento. Para la siembra, en el Ripollés (Gerona), se mataba un pollo cuya sangre se dejaba caer en el primer surco al tiempo que se decía:

\section{Aquest per Déu \\ Aquest per Sol \\ Aquest pel vent}

19 Con el término cuques también se puede estar aludiendo a lombrices, sabandijas y a bichos en general.

20 Joan AMADES, Castumari català. El curs de l'any. Volum V. Tardor (Barcelona: Salvat, $1989-1 .^{2}$ edición en $1956-$ ), p. 296. 


\section{Aquest per la pluja \\ I aquest per la llavor ${ }^{21}$.}

En el Penedés (Tarragona), se mataba una gallina o un pato, ànec, dejando caer su sangre en el primer surco, a la vez que se implorabav:

Aquest per Déu, aquest per la Venge,

aquest pel sol, aquest pel vent,

aquest per la pluja, aquest pel sembrador

$i$ aquest per la llavor 22.

En Senfrons, en la Plana de Vic (Barcelona), el rito se halla absolutamente cristianizado. El sembrador lanzaba cuatro puñados de grano, tres hacia atrás, sobre su espalda y el cuarto a la tierra, a la vez que cantaba:

\section{El primer per Nostre Senyor \\ El segon per la Mare de Déu \\ El tercer per l'Esperit Sant \\ El quant pel bons cristians ${ }^{23}$}

Como se aprecia, la distribución de la semilla entre varios seres o elementos es también fundamental y la inclusión en el reparto de la niebla, o el sol, la lluvia y el viento parece remitir a una mentalidad que considera a los elementos naturales como seres que "necesitan. su parte de la producción agrícola, en la que intervienen directamente. La muerte de un animal que, casi con toda seguridad, sería la comida de ese día nos puede poner en la pista de que ésta no sería su misión originaria ${ }^{24}$. Además, si tenemos en cuenta que el gallo - aunque aquí se mencionen pollos, gallinas y patosha sido un animal vinculado a ritos de siembra o fertilidad, debido a su poder fecundador, vemos que la elección de ese animal no es casual ${ }^{25}$.

21 Olivier de MARLIAVE, Trésor de la mythologie pyrénéenne (Toulouse: Annales Pyrénéennes, 1989), p. 286, citando a E. Busquets i Molas, aunque la página no se corresponde con el texto ni con la procedencia, ya que en ésta aparece la que ofrecemos a continuación.

22 Esteve BUSQUETS I MOLAS, Oracions, eixarms $i$ sortilegis (Ripoll: Edicions Maideu, amb la col.laboració d'Edicions Terra Nostra, 1985), p. 95. El derramamiento de la sangre de una gallina sobre el primer surco lo documenta también J. AMADES, op. cit. en nota 20, en la comarca gerundense del Empordà.

23 J. AMADES, op. cit. en nota 20, p. 295.

24 La comida ritual del gallo está atestiguada en Sarthe (Francia), donde los trabajadores la exigían a la mujer del propietario de la tierra sembrada, tal como anota Arnold VAN GENNEP, Manuel de folklore français contemporain. Tome 1. 6 Céremonies agricoles de l'automne (Paris: Picard, 1982), p. 2792.

25 José María DOMinguez MORENo, Cultos a la fertilidad en Extremadura (Mérida: Editora Regional de Extremadura. Junta de Extremadura, 1987). en la p. 22 menciona un 
Con posterioridad al análisis de los datos mencionados hasta ahora, y al leer los trabajos de P. Sébillot sobre la flora de Francia, A. van Gennep sobre las ceremonias agrícolas de otoño en Francia, y de E. Laoust sobre los bereberes, pudimos comprobar que nuestra hipótesis tiene un mayor fundamento documental y que el ámbito geográfico se amplía considerablemente.

P. Sébillot recoge una costumbre asociada a la siembra de guisantes tal como se practicaba en las cercanías de Liège (Bélgica). La sembradora - una mujer anciana - echaba un puñado hacia su espalda, diciendo: «Pô l' bon Dieur, otro en el camino "Pô l' Sint' Viengex, uno más «Pô Sin Pire"; un cuarto «Pô les mobon", es decir, para los gorriones; y, finalmente, un quinto «Pô mi et à s't elle là j'vos défin de toucher. El rito muestra el hecho, ya visto en Cataluña, de arrojar un puñado hacia atrás junto a la mención expresa de que los pájaros no deben tocar la parte que el sembrador se reserva para sí, y la inclusión de San Pedro, patrón de la localidad, en el reparto. Este mismo autor anota que en Hesbaye, localidad cercana a Liège, antes de empezar a sembrar se arrojaba un puñado grande de grano, diciendo «Pô lè mohon"; con lo cual se evitaba el futuro saqueo del sembrado por parte de los pájaros ${ }^{26}$.

Los datos que aporta Van Gennep son muy breves, pero nos ayudan a resaltar la extensión de la ritualización de arrojar el primer puñado de grano, que no parece haber estado muy difundido en Francia. De los pocos ejemplos que ofrece, hay uno procedente de la Creuse, en Champagnat (cantón de Bellegarde), que es semejante a los comportamientos vistos. El sembrador, antes de comenzar su labor, arrojaba tres puñados de grano sobre el camino, diciendo: „pour les oiseaux al lanzar el primero, pour les malhereux en el segundo y, finalmente, pour le bon Dieu, en el tercero. La costumbre tiene un elevado número de paralelos en Alemania y en los países Escandinavos, que fueron recogidos por Jahn, quien veía en esta práctica reminiscencias de un sacrificio a Odin, interpretación que es negada por Van Gennep ${ }^{27}$. Coincide la costumbre francesa con algún aspecto de las gallegas ya vistas. En el reparto se altera el orden, pero se incluye a los pájaros y a Dios, a la vez

rito que vincula al gallo con la siembra, la siega y el segador que corta la última gavilla. Este trabajador ganaba los espolones de un gallo, los cuales tenía derecho a lucir en el sombrero y, en caso de ser sembrador ese año, proporcionarían una abundante cosecha siempre que los lanzara junto al grano; el gallo era comido por todos los segadores.

26 En Bretana, el sembrador recita una oración de veinte versos al lanzar el último puñado, prometiendo una limosna para los pobres si la cosecha es buena; al no incluir el texto, no podemos decir si esta oración tiene algún paralelo con los ejemplos citados y si se cita a los pájaros de alguna forma. Todos estos datos están en Paul SÉBnLot, Le folklore de France. La Flore (Paris: Imago, 1985), p. 121.

27 A. VAN GENNEP, op. cit. en nota 24, p. 2791. No hemos podido consultar el trabajo mencionado de Jahn. 
que aparecen los malbereux en lugar de los ladrones. No queda claro si con el uso de este término hay que entender que se ofrece una parte a los necesitados, o no. El hecho de arrojar las semillas en el camino, al igual que ocurría en el caso gallego en que se lanzaban fuera de la zona productiva, parece obligatorio, ya que al destinar estos granos a divinidades, personas (distintas al propietario) o animales, se supone que no deben caer en el campo que se va a sembrar inmediatamente y cuya cosecha es lo que se pretende proteger, precisamente, ofreciendo como tributo los primeros granos lanzados ${ }^{28}$.

Por su parte, E. Laoust narra varios ritos de protección del cereal una vez que está creciendo, que se realizan entre la población bereber de Marruecos y en los que podemos ver un reparto similar al que venimos analizando. La costumbre es más compleja que lo ya visto y en oposión a ello no se lleva a cabo durante la siembra, si no que tiene lugar a mediados de febrero. Tal como ocurre en Timgissin el rito es el siguiente: se hace una cuestación -quête- en el ighrem en la que se obtiene trigo, cebada, maíz, huevos, miel, mantequilla y aceite en pequeñas cantidades, que se colocan en un plato que es llevado hasta los campos por una anciana devota rodeada por un cortejo de personas; los hombres tiran sin cesar salvas con sus escopetas, mientras que las mujeres van cantando:

-He aquí la tomzel! Que Dios preserve los campos de todo daño!

He aquí vuestra parte, oh pequeños pájaros y también la vuestra, oh jnun!r.

El cortejo se dirige hasta un bosquecillo de palmeras donde la anciana deposita la ofrenda de granos, diciendo: -He aquí vuestra parte del banquete, oh jnun y pequeños pájaros”. Finalmente se regresa al pueblo entre tiros de fusil ${ }^{29}$. El ritual tiene paralelos en el sur de Marruecos y enlaza con otros semejantes de protección frente a elementos peligrosos. Para este autor los pájaros son la encarnación de los malos espíritus que se pretende echar y como otros muchos ritos de expulsión" suelen aparecer asociados a la ce-

28 En la parábola del sembrador -que recogen los evangelios de S. Mateo (13, 1-9), .S. Lucas $(8,4-8)$ y S. Marcos $(4,1-9)$-, éste arroja granos que caen en el camino y son comidos por los pájaros, pero no se puede deducir de su lectura que se esté describiendo algún tipo de rito similar a los que venimos analizando.

29 Emile LAOUST, Mots et choses berbères (Rabat: Société Marocaine d'Edition, 1983; facsímil de la primera edición, de 1920), p. 340. El autor da los textos transcritos del árabe y la traducción francesa, por lo que optamos por traducir ésta última. Las ofrendas a los pájaros se documentan en la tradición francesa asociadas a pastores y boyeros en relación con la ayuda que reciben de las urracas para advertirles de la llegada del lobo; en el Poitou se les daba una crêpe el martes de carnaval, la cual era colocada en las ramas altas de un árbol, tal como anota Paul SÉBLLOT, Le folklore de France. La Faune (Paris: Imago, 1984), pp. 197-198. 
lebración de fiestas islámicas; es decir se integran en el calendario religioso musulmán a pesar de ser comportamientos de tradición preislámica ${ }^{30}$.

Sin poder negar absolutamente que los pájaros sean representativos del mal, creemos que no es ésta la correcta interpretación de la costumbre reseñada, aunque sí puede serlo de otras que narra este autor y en las cuales se mata a uno de estos animales. A la luz de los materiales que hemos acumulado pensamos que la actitud hacia los pájaros implica un cierto respeto que choca con otros ritos en los que el tratamiento que reciben es radicalmente diferente; en éstos se puede apreciar un carácter fundamentalmente intimidatorio en las fórmulas que se dicen y por las cuales se les conjura para que no dañen el grano. Casualmente, también son gallegos los dichos que hemos documentado en España y que se dirigen a los animales dañinos - con una carga simbólica maléfica- y a las brujas en el Sábado Santo. Los conjuros suelen acompañarse de rezos y asperjamientos con agua bendita que es esparcida con ramitas de olivo, xesta o laurel, las cuales quedarán plantadas en el campo. Como vemos, al igual que ocurría en la costumbre bereber, también se eligen fechas de marcado carácter religioso para llevarlas a cabo. En Mourente (Pontevedra) se dice:

\author{
Cobras e sapos \\ bruxas e ratos, \\ toupas e meigas \\ fóra das miñas leiras
}

Mientras que en Xavestre (La Coruña), la imprecación es más genérica e incluye al agua bendita como elemento fundamental:

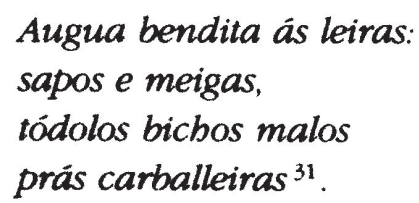

Dirigidas concretamente contra los pájaros hay varios ritos y fórmulas portugueses, que recogió Leite de Vasconcellos, y en los que se les conmina a abandonar los campos en la siembra. En Minho, para librar al milho alvo o al panizo de los pájaros, ponen hiel de un suino en una vasija nueva, no lavada, que, junto con un ramo de castaño y una piedra en el sobaco, es llevada por una mujer que debe dar tres vueltas al campo; además, esparce tres puñados de arena, diciendo:

\footnotetext{
30 E. LAOUST, op. cit. en nota anterior, pp. 340-350 y en concreto su nota 1 que empieza en la p. 345.

31 Clodio González Pérez, A festa dos Maios (Vigo: Ir Indo Edicións, 1989), pp. 22-23.
} 
Pardejos ide ao monte, que ten mel,

Deixai o milho alvo, que tem fel

La vasija es enterrada posteriormente, teniendo en cuenta que no se debe espantar a los pájaros y que la hiel tendrá que ser retirada antes de segar el cereal ${ }^{32}$.

En el concejo de Guimarães, se entierra la hiel diciendo:

Passarinhos, ao monte, ao monte,

Que o meu campo ten fel.

$O$ do meu vizinho tem mel $^{33}$.

En Portugal - sin mayor precisión- se entierra un cántaro con hiel de buey durante la noche en el campo que se quiere librar de los pájaros y una persona, hombre o mujer, en faldra de camisa da una vuelta al campo diciendo:

\author{
Passarinhos deixai o meu painço \\ Que tem fel! \\ Ide para o monte \\ Que tem mel!
}

La hiel, al igual que ocurría en el caso anterior, deberá quitarse cuando se aproxima la siega ya que, en caso contrario, el cereal se volvería amargo ${ }^{34}$.

32 J. LEITE DE VASCONCELlos, Etnografia portuguesa. Tentame de sistematização. Volume $V$. Organizado por $M$. Viegas Guerrero con la colaboração de Alda da Silva Soromenho e Paulo Caratão Soromenho (Lisboa: Imprensa Nacional. Casa da Moeda, 1982), pp. 668-669.

33 J. LEITE DE VASCONCELlOS, op. cit. en nota anterior, p. 669.

34 J. LEITE DE VASCONCELLOS, Tradições populares de Portugal. Organização e apresentação de M. Viegas Guerrero (Lisboa: Imprensa Nacional. Casa da Moeda, 1986, 2." ed. revisada y aumentada), p. 200. En la obra de este autor citada en la nota 32, menciona que en Mouril (Minho) un hombre, con la camisa fuera, debe dar la vuelta al campo llevando una vasija con hiel y diciendo una oración, para después colocar la vasija en el campo, con lo cual se obtendrá la protección frente a los pájaros en tanto que la vasija permanezca allí; como en otras ocasiones se deberá retirar antes de la siega. También se documenta la práctica de enterrar una vasija en Cataluña; en Linyola y otros pueblos de la zona de Urgell (Lérida), para asegurar el éxito de la siembra, evitar que los pájaros se comieran el grano y alejar a los animales roedores, se enterraba un sapo vivo dentro de un cántaro J. AMADES, op. cit. en nota 20 , p. 291. Según P. SÉBLLơ, op. cit. en nota 26, en el Périgord (Francia), para impedir que los pájaros coman el grano cuando el trigo ha espigado, también se mete una rane de buisson en una vasija de barro y se entierra en mitad de la parcela. Este tipo de prácticas tiene precedentes clásicos y medievales a los que hemos aludido en un trabajo nuestro, sLa necesidad de una visión etnológica en los estudios arqueológicos. El mundo agrícolan, IV Congreso de Arqueología Medieval Española (Alicante: e.p.). 
Una variante del mismo ritual se da en Cinfães, en donde hay que dar tres vueltas al campo con una panela colocada con la boca hacia el pecho y decir:

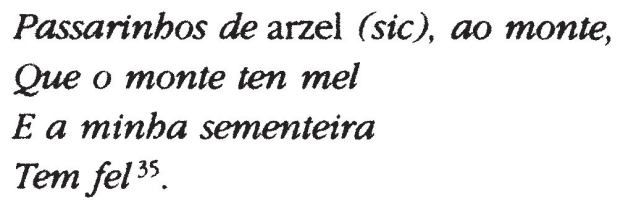

Pensamos que la correcta explicación del tratamiento respetuoso hacia los pájaros se encuentra en muchas creencias que existen en torno a ellos. Acudiendo a trabajos de J. Amades, podemos ver con claridad cuál era la situación en Cataluña. Por un lado, vemos cómo los pájaros juegan un papel importante en relación con la siembra; este autor mencionó que en varios puntos del país se creía que los pájaros traían la semilla al campo y que, en ocasiones, se pensaba que la transportaban desde el cielo. En el Pla de Barcelona se decía que los pájaros cogen su atributo" de grano por haber sido portadores de la semilla y, de una manera vaga, se opinaba que el canto y el vuelo de los pajarillos ayudaba a la siembra ${ }^{36}$. En Reus (Tarragona), se contaba que la pastorella fue llevada al Niño Jesús por los pastores, siendo su primer juguete; por esto es un pájaro bendito y es pecado matarle; además, Dios quiso que siguiera a los sembradores y labradores para comerse los gusanos, evitando así que estos animales acabaran con el grano ${ }^{37}$. El papel positivo de los pájaros hacia el grano aparece en otra narración que tiene como protagonista a la cogullada. Este ave es la encargada de trasmitir al sol la situación del trigo, indicándole si ya está maduro y listo para la siega; para ello le lleva una espiga con el fin de que el sol pueda ver cómo se encuentra de sazonado. A pesar de que el sol reprende su comportamiento el ave lo repite cada año, con la excusa de que:

Per un bri, per un bri que $m$ 'ban de dir 38 .

35 J. LeTtF: DE VASCONCELLOS, op. cit. en nota anterior, p. 200.

3. J. AMADES, op. cit. en nota 20, pp. 288-289 y 290. Quizá haya que poner en relación la idea de que los pájaros traen la semilla, con una narración que recoge este autor en otra de sus obras, y en la cual se explica el por qué de la ausencia de la cola del cuco. En ella se dice que este pájaro y la urraca se asociaron para sembrar un campo de trigo; el texto se encuentra en Joan AMADEs, L'origine des bêtes. Petite cosmogonie catalane (Carcassonne: Garae / Hesiode, 1988), en p. 87; el libro traduce parte del Folklore de Catalunya. Rondallística (Barcelona: Selecta, 1950), que no tenemos a mano en este momento.

37 J. AMADES, op. cit. en nota 36 en segundo lugar, pp. 74-75.

38 J. AMADES, op. cit. en nota 36 en segundo lugar, p. 65. 
Si analizamos otras narraciones relativas a los pájaros, en las cuales se trata de explicar el porqué de sus características o de la interpretación de su canto, podemos apreciar que estos tienen actuaciones positivas hacia personajes como Jesús o la Sagrada Familia, lo cual conduce a que se recompensen sus comportamientos de varias formas, alguna de las cuales consiste en conceder un estatus privilegiado que implica la imposibilidad de que el animal sea perseguido y muerto ${ }^{39}$. En otros casos se constata la creencia de que algunos pájaros han sido creados por Dios, frente a otros que lo han sido por el diablo, lo que conduce a la existencia de pájaros buenos y pájaros malos y a actitudes diferentes ante ellos por parte de las personas ${ }^{+0}$.

Existe una costumbre vasca que asocia el pan a los pájaros, aunque sin relación directa con la siembra o el cereal, que se ha documentado en Zerain (Guipúzcoa). Era normal que si sobraba un trozo de pan en la merienda, se dejara éste sobre una piedra, tras haberlo besado, diciendo: Txoriak-jan dizutela, o Aingeruak bedeinkatu eta txoriak jan zaitela (Que te bendigan los ángeles y te coman los pájaros) ${ }^{41}$. Quizá aluda a algo similar el denominado pa de moixons, pan de los pajaritos, que mencionó Violant i Simorra sin explicar de qué se trataba ${ }^{42}$.

En relación con la cosecha, P. Sébillot, indicó que en Francia -n concreto en Léon- el oir el canto de la lechuza cuando se llega a un campo que se va a labrar es signo de la obtención de una buena cosecha ${ }^{\text {t3. }}$. Este autor menciona también cómo ciertas aves aparecen asociadas al comienzo de la siembra, ya que con su canto indican que es el momento oportuno de efectuarla ${ }^{44}$; creencia que podría enlazar, en cierta medida, con alguna de las catalanas citadas.

39 También el gorrión es un pájaro bendito ya que, según una narración de Ripoll (Gerona), sacó una astilla al niño Jesús cuando jugaba en el taller de su padre. En el Pla de Cabra (Tarragona) el motivo de la bendición se debió a que el gorrión fue a prevenir a Cristo de la traición de Judas; esta tradición tiene paralelos en Sicilia y Wallonia; J. AMADES, op. cit. en nota 36 en segundo lugar, pp. 145-146 y 146, respectivamente.

40 J. AMADES, op. cit. en nota 36 en segundo lugar, pp. 51-53. La tradición tiene paralelos en Francia.

41 José Miguel de Barandiarán y Ander Manterola (direcc.), La alimentación doméstica en Vasconia (Bilbao: Eusko Jaurlaritza. Etniker Euskalerria, 1990), 755 pp. Atlas etnográfico de Vasconia. Tomo I, p. 147.

42 Ramón VIOLANT i SIMORRA, La induistria casolana del pa al Pallars Sobirà. El gra. Els molins. Els forns. Pastar. Costums $i$ creences (Barcelona, 1936) y en Obra oberta, 2 (Barcelona: Alta Fulla, 1979), pp. 67-161, en concreto en p. 155.

43 P. SÉBILLot, op. cit. en nota 26, p. 119.

44 Es posible documentar este tipo de información en Francia desde la Edad Media, ya que en el Roman de Renart se dice: 
El papel benefactor de algunos pájaros fue apuntado, asimismo, por Sébillot, quien recoge una fórmula dirigida a la alondra, adjudicándole la función de intermediaria ante Dios para la obtención de un buen tiempo y una buena cosecha:

\author{
Alouette du printemps, \\ Prie l'bon Dieu qu'il fasse beau temps \\ Pour faire pousser du bon froment, \\ Pour faire du bon pain blanc, \\ Pour tous ces petits enfants ${ }^{45}$.
}

Lamentablemente, no tenemos datos semejantes para otras zonas de España que nos ayuden a precisar la importancia de este tipo de visión sobre los pájaros, en las áreas donde se han practicado los ritos del reparto de los primeros puñados de grano en la siembra ${ }^{46}$. Esta falta de documentación no quiere decir, obviamente, que no exista en la realidad este comportamiento. Como hemos visto, no obstante, la presencia de la actitud respetuosa no excluye la posibilidad de que se dé la contraria, es decir, que se considere a los pájaros como animales dañinos y se les aleje por todos los medios posibles, llegando incluso hasta su eliminación física.

\title{
J'ai oï les grues chanter \\ Qui nos tesmoignent par raison \\ Que de semer avons saison
}

Y la misma actitud se halla en el Poitou, donde el canto de la grulla, al pasar por primera vez, es 'traducido" como: sème ton blé, paresseux! Quand i' retorn'ai, i' en mangerai mon saoul, P. SÉBlLLot, op. cit. en nota 29, p. 194.

45 P. SÉBillot, op. cit. en nota 29, pp. 198-199. La misma fórmula, con una insignificante variación, es dicha por los niños del Orléanais; ver Jean BAUCOMONT, ‘Les formulettes enfantines d'incantation", Arts et Traditions Populaires, XIII, 3-4 (1965): 243255, en p. 246. En este artículo - p. 247- se puede ver la asociación del pan a diversos animales a través del ofrecimiento infantil a cambio de información sobre el buen tiempo (con la mariquita), o sin ningún tipo de contrapartida (con el murciélago).

46 En Galicia se documenta la creencia de que las almas pueden aparecer transformadas en pájaros. Por la relación que tiene con la siembra y el trabajo agrícola, conviene reseñar una aparición que cita Vicente Risco, .Etnografia. Cultura espiritual, en Historia de Galiza, dirixida por Ramon Otero Pedrayo (Madrid: Akal, 1979), p. 419, ya que se realizó ante un labrador que estaba arando y el pájaro se posó en el yugo de los bueyes pidiendo que dijeran una misa por su alma. Este mismo autor, en pp. 385 y 360 , menciona la costumbre de llevar pájaros enjaulados a las iglesias para que las alegraran con su canto; en concreto menciona que esta práctica se llevaba a cabo durante la novena del Santísimo Cristo en Orense. 
OTROS COMPORTAMIENTOS ANTE LA PROTECCIÓN DE LOS SEMBRADOS FRENTE A LAS AVES Y DEMÁS ANIMALES DAÑINOS

Sin salimos del ámbito occidental, podemos recurrir a testimonios históricos, de época romana o tardorromana, en los que se ven actitudes diferentes y similares de protección del grano frente a pájaros y hormigas. Ovidio, en sus Fastos recoge un ruego a Tellus y a Ceres, como responsables del crecimiento de las plantas, en el que, entre otras cosas, pide:

Cuando sembramos, despejad el cielo con vientos apacibles; cuando la semilla está enterrada, regadla con la lluvia de los cielos. Impedid que los pájaros, ruina de los cultivos, devasten los campos de trigo en destructoras bandadas. También vosotras, hormigas, dejad en paz los granos enterrados: después de la cosecha la abundancia de vuestro botín será mayor [...] Estas son las peticiones que formulo; esto es lo que también vosotros, campesinos, debéis pedir ${ }^{47}$.

A pesar de que el fin último es que los pájaros y las hormigas no ataquen el grano recién sembrado, existe una diferencia clara en la actitud ante los distintos animales, ya que mientras en los casos españoles, franceses, belgas y marroquíes mencionados se aprecia un respeto hacia ellos o, al menos, un cierto temor a su actuación que se conjura directamente con las prácticas descritas, en el caso latino se invoca a los dioses para que actúen como protectores.

El comportamiento respetuoso hacia los animales y la ofrenda de una parte del grano sí que se puede documentar en un texto del siglo vi sobre las costumbres paganas del noroeste hispano; nos referimos al conocido De correctione rusticorum de San Martín de Braga. La intención del obispo es, como se sabe, atacar una serie de costumbres consideradas paganas, que se encuentran extendidas entre sus feligreses; entre éstas menciona un cierto culto a los ratones y a las polillas:

Por otra parte ¿qué puede dolorosamente decirse de aquel error tan craso, de que se celebren los días de las polillas y de los ratones y, si está permitido decirlo, de que el hombre cristiano venere como a un dios a ratones y polillas? Creen que si no se les ofrece, para salvaguarda del tonel o de la arquita, pan o paño, no respetarán de ninguna manera nada de lo que encuentren en pago de los obsequios que se les han tributado [...] Resulta que practicais estos inútiles actos supersticiosos ocultamente o en público y no dejais nunca de hacer sacrificios a los demonios

47 La traducción es de M. A. Marcos Casquero, en Publio Ovidio Nason, Fastos (Madrid: Editora Nacional, 1984), p. 147.

48 Que corresponde al texto latino: Iam quid de illo stultissimo errore cum dolore dicendum est, quia dies tinearum et murium observant et, si dici fas est, bomo Christianus pro deo mures et tineas veneratur? Quibus si per tutelam cupelli aut arculae non subducantur aut panis aut pannus, nullo modo pro feriis sibi exbibitis, quod invenerint, 
R. Jove Clols, en el aparato crítico que acompaña a la edición de este texto, menciona a Ovidio y a las Paganalia, fiestas que se celebraban en enero y de las que procede el texto citado anteriormente, pero no acaba de encontrar la unión de ambos hechos e incide en la dificultad de interpretación del pasaje de San Martín. A pesar de que la relación no es ni mucho menos directa, sí creemos que las costumbres actuales ayudan a entender la mentalidad existente en las paganas: cómo se intenta controlar la actuación de los ratones en un caso y de los pájaros y hormigas en otro, para que no interfieran en la productividad de las cosechas a cambio de ofrecerles una cierta cantidad de grano. No se puede asegurar que el rito al que alude el Dumiense fuera similar al que se ha documentado en los años cincuenta en Galicia, pero tampoco parece muy arriesgado suponer que tuviera algún contacto con él.

\section{CONCLUSIÓN}

Creemos que la simple exposición de los hechos es suficientemente clara como para mostrar, sin lugar a dudas, que las actuaciones milagrosas asociadas a San Isidro y la Virgen de Valvanera tienen su raíz en la reinterpretación - iante la falta de comprensión? - de rituales de siembra del primer grano.

La consideración positiva de que gozan ciertos pájaros explicaría parcialmente el porqué del comportamiento campesino. A ello se debe añadir la importancia que supone el momento de iniciar la siembra y, por tanto, los trabajos que conducirán a la obtención del futuro sustento; lo cual llevaría a la ritualización de algunos actos. Frente a esto, el peligro real que suponen las aves para la cosecha hace que puedan no comprenderse estos ritos y que traten de explicarse como productos de una "persona loca" y ajenos a thombre de buen seso", por decirlo con las palabras aplicadas a San Isidro.

La variedad de actitudes ante pájaros, hormigas y ratones, y ante aquel a quien debe ofrecerse parte de la cosecha, muestra aspectos semejantes en ciertas ocasiones y opuestos en otras, a lo largo del tiempo y del espacio. Pero, sea a través del halago o de la intimidación, el campesino ha recurrido hasta nuestros días a prácticas que tendieran a asegurarle una buena cosecha más allá de la imprescindible realización material del trabajo agrícola.

\section{JOSÉ LUIS MINGOTE CALDERÓN Museo Arqueológico Provincial Palencia}

\footnotetext{
parcent [... J Ecce istas superstitiones vanas aut oculte aut palam facitis, et nunquam cessatis $a b$ istis sacrificiis daemonum. Ambos se encuentran en Rosario Jove Clols, Sermón contra las supersticiones rurales (Barcelona: El Albir S.A., 1981), pp. 33 y 32, respectivamente.
} 
En este artículo se analiza un milagro realizado por San Isidro que se halla en textos cultos a partir del siglo xvII. La explicación que en él se da del ofrecimiento a los pájaros y las hormigas de una parte del grano destinado a la siembra, debe ser considerada como una interpretación católica de un ritual asociado a la siembra del primer puñado de grano. Esta costumbre aparece claramente documentada en los estudios etnográficos españoles, así como en los de ámbitos próximos: Europa occidental y norte de África.

This paper focuses on a miracle achieved by St. Isidro which is found in learned texts from the xvith century. The explanation of the offering of part of the sowing grain to birds and ants should be considered as the Catholic interpretation of a ritual associated with the sowing of the first handful of grain. This custom is well documented in Spanish ethnographic studies as well as in those from West Europe and North Africa. 